\title{
The dynamical Casimir effect in a periodically changing domain: a dynamical systems approach
}

\author{
Nikola P Petrov \\ Department of Mathematics and Michigan Center for Theoretical Physics, University of \\ Michigan, Ann Arbor, MI 48109-1109, USA
}

Received 31 July 2004, accepted for publication 14 October 2004

Published 1 March 2005

Online at stacks.iop.org/JOptB/7/S89

\begin{abstract}
We study the problem of the behaviour of a quantum massless scalar field in the space between two parallel infinite perfectly conducting plates, one of them stationary, the other moving periodically. We reformulate the physical problem into a problem about the asymptotic behaviour of the iterates of a map of the circle, and then apply results from the theory of dynamical systems to study the properties of the map. Many of the general mathematical properties of maps of the circle translate into properties of the field in the cavity. For example, we give a complete classification of the possible resonances in the system, and show that small enough perturbations do not destroy the resonances. We use some mathematical identities to give a transparent physical interpretation of the processes of creation and amplification of the quantum field due to the motion of the boundary and to elucidate the similarities of and the differences between the classical and quantum fields in domains with moving boundaries.
\end{abstract}

Keywords: dynamical Casimir effect, dynamical systems, wave equation, geometrical optics, resonances

\section{Introduction}

Recently the problem of the behaviour of the fields in a cavity with a (periodically) moving boundary has received significant attention. From a mathematical point of view, it constitutes an instructive example of a parametrically driven system that exhibits interesting resonant effects. From the point of view of physics, besides its fundamental importance as a modification of the Casimir effect, it can be used as a model for the mechanism of formation of wavepackets in lasers, processes in atomic physics, and even interstellar flight [49]! We would like to draw the reader's attention to the reviews of the Casimir effect by Bordag et al [4], the books by Milton [53], and Mostepanenko and Trunov [55], and to the recent review by Dodonov [22] devoted specifically to the dynamical (or nonstationary) Casimir effect (i.e., the quantum effects in a pulsating cavity) which contains more than 300 references.

In this paper we will apply a method that we have developed in [47] (and generalized in [58]) to study the behaviour of the classical electromagnetic field in a one- dimensional cavity with a moving wall by using the methods of the theory of dynamical systems. Here we will employ this methodology to analyse the quantum problem. Our approach is based on studying the collective behaviour of the characteristics of the wave equation by applying the theory of circle maps. The general theorems allow us to predict the behaviour of the system without solving partial differential equations. We show that the mechanism of the resonant amplification of the quantum field is the same as in the classical case-it is due to the Doppler effect at reflection from the moving mirror. In the quantum case, however, the motion of the mirror 'creates' new field which is then amplified by the Doppler effect. Using some mathematical identities, we give a simple physical interpretation of the different contributions to the energy density.

In the rest of the introduction, we review very briefly some of the literature related to the mathematical and physical aspects of our approach, referring the reader to the review [22].

The mathematical theory of the solutions of the wave equation in the presence of (periodically) moving boundaries 
in one or more spatial dimensions has been developed by Cooper [7-9], Cooper and Koch [10], Yamaguchi and collaborators [63, 64, 66, 65], Dittrich et al [19]. In the physics literature, the classical version of the problem was studied by Dittrich et al [20], Cole and Schieve [6], Mèplan and Gignoux [52], who used the geometric method of solving the wave equation (i.e., the method of characteristics), and recognized that in some cases the field develops wavepackets that become narrower with time.

The foundations of the quantum theory of the problem of a one-dimensional resonator with a moving wall were laid by Moore [54], and developed by Fulling and Davies [30, 14]. Dodonov et al $[25,26,24]$ considered the case of resonant motion of the mirror within Moore's formalism; they and Jaekel and Reynaud [36], Méplan and Gignoux [52], and others predicted that the force between the mirrors can be enhanced significantly in the resonant case.

In [44], Law proposed an exact analytic solution for a particular choice of the motion of the mirror, for which the field in the cavity develops two wavepackets which become narrower in time and whose energy grows. Law's solution was generalized by Wu et al [62], who constructed motions of the mirror for which the field develops several wavepackets.

Dodonov [21] noticed that small enough 'detuning' from the exact resonance conditions does not change the qualitative features of the behaviour of the field in the cavity. Within our approach, one can find explicitly for what detuning the behaviour of the field will change dramatically. The packet formation in a resonantly pulsating resonator was studied in detail by Dodonov and Andreata [23, 2].

Ideas from dynamical systems (i.e., study of the iterates of a certain map) have been used by Cooper $[8,9]$ (maps of the interval), Méplan and Gignoux [52] (area-preserving maps of the cylinder), Dittrich et al [20, 19], Yamaguchi [63, 64, 66] (circle maps).

\section{The method of characteristics and dynamical systems}

In this section, we explain the physical set-up, pose the mathematical problem, and explain how to analyse it. We recommend that the reader consult our paper [47] for details.

We will refer often to the electromagnetic (EM) field in the cavity (meaning the classical, not quantum, field); as it turns out, some aspects of the problem are similar in the classical and in the quantum case.

\subsection{Description of the physical system}

Consider the EM field in the empty space (no medium, electric charges, or currents) between two parallel perfectly reflecting mirrors, one stationary at $x=0$, the other moving according to $x=a(t)$. The function $a$ must satisfy the physically natural conditions $a(t)>0$ for each $t$ (the resonator never collapses to zero length), $\left|a^{\prime}(t)\right|<1$ (the speed of the moving mirror never exceeds the speed of light). To avoid technicalities, we assume that $a(t)$ is a smooth $\left(C^{\infty}\right)$ function. We will focus on the case in which the motion of the mirror is 1-periodic (i.e., periodic of period 1 ):

$$
a(t+1)=a(t) \quad \text { for each } t .
$$

Examples of such functions are

$$
\begin{aligned}
a(t) & =\frac{\alpha}{2}+\frac{\beta}{2 \pi} \sin 2 \pi t, \quad \frac{\alpha}{2}>\frac{|\beta|}{2 \pi}, \quad|\beta|<1 ; \\
a(t) & =\frac{\alpha}{2}+\frac{\beta}{2 \pi} \sin \left(2 \pi t+\gamma(\sin 4 \pi t)^{2}\right), \\
\frac{\alpha}{2} & >\frac{|\beta|}{2 \pi}, \quad|\beta|(1+2|\gamma|)<1 .
\end{aligned}
$$

In our numerical simulations we will use the function $a(t)$ from (2).

\subsection{The boundary value problem}

Since there are no charges and currents in the cavity, we impose Coulomb gauge $A_{0}=0, \nabla \cdot \mathbf{A}=0$ on the EM 4-potential $A_{\mu}=\left(A_{0}, \mathbf{A}\right)$, and obtain that $\mathbf{A}$ satisfies the wave equation. We consider linearly polarized plane waves propagating in the $x$ direction, so we can assume without loss of generality that the vector potential has the form

$$
\mathbf{A}(t, x)=A(t, x) \mathbf{e}_{y} .
$$

The function $A(t, x)$ satisfies the $(1+1)$-dimensional wave equation

$$
A_{t t}(t, x)-A_{x x}(t, x)=0
$$

in the spatio-temporal domain $\Lambda:=\left\{(t, x) \in \mathbb{R}^{2} \mid 0<x<\right.$ $a(t), t>0\}$. The boundary conditions (BCs) come from the fact that in the coordinate frame instantaneously co-moving with the mirror, the component of the electric field tangential to the mirror must vanish at the mirror, which yields the "perfect reflection' $\mathrm{BCs}$

$$
A_{t}(t, 0)=0, \quad A_{t}(t, a(t))+a^{\prime}(t) A_{x}(t, a(t))=0
$$

for each $t \geqslant 0$. Geometrically, the BCs (4) mean that the derivative of $A(t, x)$ along the world line of the mirror (i.e., the line $\{(t, a(t)) \mid t \in \mathbb{R}\}$ in the space-time diagram) must be 0 . Note that the Dirichlet BCs

$$
A(t, 0)=c_{1}=\mathrm{const}, \quad A(t, a(t))=c_{2}=\mathrm{const}
$$

are equivalent to (4). Parenthetically, we would like to note that, in our simple one-dimensional model, Neumann BCs are not Lorenz covariant, so they are not physically natural for the case of EM fields. Neumann BCs, however, have been studied for nonrelativistic motion of the mirror in the more realistic three-dimensional case $[50,56,11,1]$. If one imposes Neumann BCs in the one-dimensional case, the predictions of the theory are dramatically different from those of the "perfect reflection' BCs (4) (see [58, section 5.6] or [19, section 4]).

In the quantum case [54], one obtains the boundary value problem consisting of equation (3), the Dirichlet BCs (5) with $c_{1}=c_{2}=0$, and initial conditions

\subsection{The method of characteristics}

In the absence of spatial boundaries (i.e., if $\Lambda=\{(t, x) \in$ $\left.\mathbb{R}^{2} \mid t>0\right\}$ ), the solution of the wave equation (3) with 'perfect reflection' (4) or Dirichlet (5) BCs, and initial conditions $A(0, x)=\psi_{1}(x), A_{t}(0, x)=\psi_{2}(x), x \in \mathbb{R}$, is a superposition of waves propagating to the left and to the right: $A(t, x)=$ 


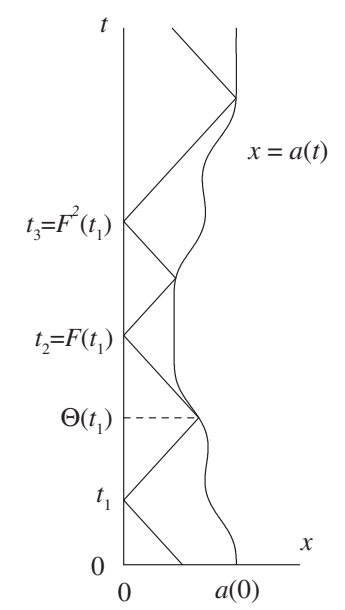

Figure 1. Characteristics of the wave equation.

$\Psi^{+}\left(x_{0}^{+}\right)+\Psi^{-}\left(x_{0}^{-}\right)$, where $x_{0}^{ \pm}=x \pm t$, and $\Psi^{ \pm}(s)=\frac{1}{2}\left[\psi_{1}(s) \pm\right.$ $\left.\int_{c}^{s} \psi_{2}\left(s^{\prime}\right) \mathrm{d} x\right]$ ( $c$ is an arbitrary constant, the same for $\Psi^{+}$and $\Psi^{-}$). Geometrically, in the space-time diagram, the waves propagate along the characteristics $x \pm t=$ const.

In the presence of spatial boundaries (stationary or moving), the characteristics are no longer straight lines, but are piecewise linear, each part of them being a straight line at $45^{\circ}$ with respect to the $t$ axis (see figure 1). In order for the BCs (4) to be satisfied, the field changes sign with each reflection, so

$$
A(t, x)=(-1)^{N_{+}} \Psi^{+}\left(x_{0}^{+}\right)+(-1)^{N_{-}} \Psi^{-}\left(x_{0}^{-}\right),
$$

where $N_{ \pm}$is the number of reflections of the corresponding broken characteristic by a mirror between the initial moment $t=0$ and the present time $t$. For a proof that this algorithm works, see [47, section II.B].

\subsection{The importance of the reflections; the Doppler effect}

Loosely speaking, the 'density' of the characteristics in the space-time diagram is proportional to the energy density of the EM field. Consider two characteristics corresponding to the ends of a narrow wavepacket. During free propagation (no reflection), the width of the wavepacket and the vector potential A do not change. At reflection from the moving mirror, however, not only does $A$ change sign, but also the width of the wavepacket changes. If the width of the wavepacket before the reflection was $\Delta$, and the reflection occurs at time $t$ (assume that the wavepacket is so narrow that the reflection happens almost instantaneously), simple trigonometry shows that after the reflection the width $\Delta^{\prime}$ of the wavepacket is $\Delta^{\prime}=\Delta / D(t)$, where

$$
D(t)=\frac{1-a^{\prime}(t)}{1+a^{\prime}(t)}
$$

is the Doppler factor at reflection at time $t$. The term 'Doppler factor' comes from the fact that the initial (classical) EM energy of the wavepacket, $\frac{1}{8 \pi} \int_{\Delta}\left[A_{t}(t, x)^{2}+A_{x}(t, x)^{2}\right] \mathrm{d} x$, increases by a factor of $D(t)$ at reflection from the moving mirror (see [58, section 2.4] for a simple proof). Clearly, if the mirror is moving inwards (outwards) at the time of reflection, the energy of the wavepacket will increase (decrease). If it happens that every time a certain group of nearby characteristics (representing a wavepacket) is reflected from the moving mirror while the mirror is moving inwards, then they are going to get closer together, which will lead to squeezing of the wavepacket (see [25] and [36]) and to exponential growth of the energy of the field, as we will see below.

Since the motion of the boundary is 1-periodic (1), the position $a(t)$ and the velocity $a^{\prime}(t)$ of the mirror, as well as the Doppler factor $D(t)(6)$, do not depend on the integer part of $t$, but only on its fractional part,

$$
\{t\}:=t-\lfloor t\rfloor,
$$

which we will refer to as the phase of the motion of the mirror. To make the phase of $t$ change continuously as $t$ increases, we will think of $\{t\}$ as belonging to a circle of length 1, i.e., to the interval $[0,1]$ with its ends identified. The long-time behaviour of the field depends on the asymptotic behaviour of the characteristics, which in turn can be analysed by invoking the mathematical theory of circle maps, as explained below.

\subsection{From characteristics to circle maps}

Since characteristics belong to a very simple class of plane curves-namely, piecewise linear at a $45^{\circ}$ angle with the $t$ axis - to reconstruct a particular characteristic, it is enough to know only one moment at which this characteristic is reflected from, say, the stationary mirror.

To study the collective behaviour of the characteristics, we introduce the time advance map $F: \mathbb{R} \rightarrow \mathbb{R}$ such that if certain characteristic is reflected from the stationary mirror at time $t_{1}$, the next reflection from the same mirror occurs at time $t_{2}=F\left(t_{1}\right)$ (see figure 1). To derive an expression for $F$ in terms of the function $a$ giving the motion of the mirror, we notice that the time $\Theta\left(t_{1}\right)$ between $t_{1}$ and $t_{2}$ at which the characteristic is reflected from the moving mirror satisfies $\Theta\left(t_{1}\right)-a\left(\Theta\left(t_{1}\right)\right)=t_{1}$, which can be written as $(\mathrm{Id}-a)\left(\Theta\left(t_{1}\right)\right)=t_{1}$; therefore

$$
\Theta=(\operatorname{Id}-a)^{-1} .
$$

On the other hand, $F\left(t_{1}\right)=\Theta\left(t_{1}\right)+a\left(\Theta\left(t_{1}\right)\right)=(\operatorname{Id}+a)\left(\Theta\left(t_{1}\right)\right)$; thus

$$
F=(\operatorname{Id}+a) \circ(\operatorname{Id}-a)^{-1} \text {. }
$$

The conditions $a(t)>0$ and $\left|a^{\prime}(t)\right|<1$ guarantee the invertibility of ( $\operatorname{Id} \pm a$ ) (and hence the existence of $\Theta$ and $F$ ) as well as the fact that $F$ is strictly increasing and, therefore, invertible. We leave it to the reader to check that $a$ can be expressed in terms of $F$ as

$$
a=\frac{1}{2}(F-\mathrm{Id}) \circ\left[\frac{1}{2}(F+\mathrm{Id})\right]^{-1} .
$$

The 1-periodicity (1) of $a$ guarantees that $F$ satisfies the property

$$
F(t+1)=F(t)+1 .
$$

Since only the phase $\{t\}(7)$ is physically important, instead of considering the function $F: \mathbb{R} \rightarrow \mathbb{R}$, we define the function

$$
f: S^{1} \rightarrow S^{1}:\{t\} \mapsto\{F(\{t\})\}
$$

that maps the phase $\{t\}$ at some reflection from the stationary mirror to the phase $\{F(\{t\})\}$ at the next reflection from the same 


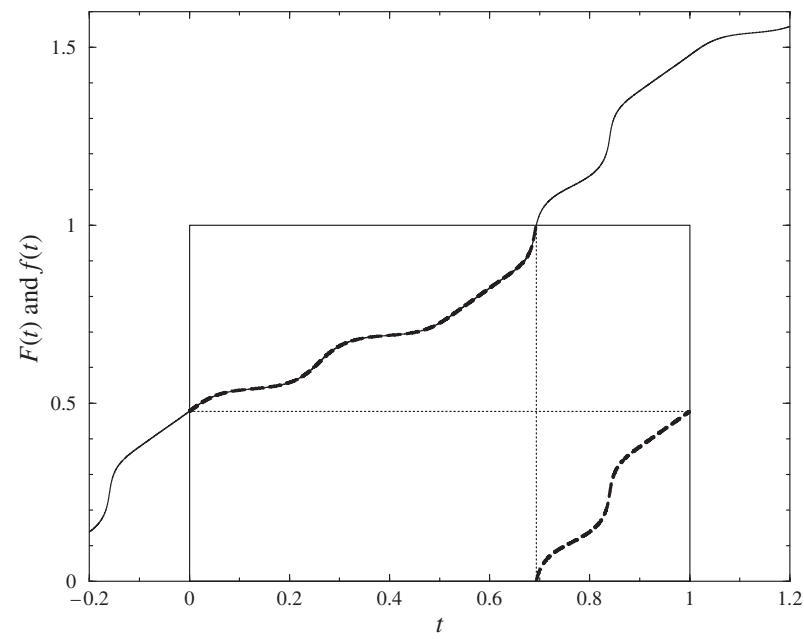

Figure 2. A graph of the functions $F$ (thin solid curve) and $f$ (thick dashed curve) corresponding to the function $a$ (2) for $\alpha=0.35$, $\beta=0.4, \gamma=0.7$.

mirror. Here $S^{1}$ stands for the 'circle', i.e., the interval $[0,1]$ with its ends identified (in mathematical notation, this can be written as $S^{1}=\mathbb{R} / \mathbb{Z}$, where $\mathbb{Z}$ stands for the integers). The function $f$ is well defined due to (10).

If the first reflection of a particular characteristic from the stationary mirror occurs at time $t_{1}$, the times of the subsequent reflections are $F\left(t_{1}\right), F^{2}\left(t_{1}\right), F^{3}\left(t_{1}\right), \ldots$, where

$$
F^{n}:=\underbrace{F \circ F \circ \cdots \circ F}_{n \text { times }}
$$

is the $n$th iterate of the function $F$. Since the asymptotic behaviour of the characteristics is completely determined by the asymptotic behaviour of the phases at reflection, the longtime behaviour of the system can be studied by analysing the high iterates of $f$. The branch of mathematics that studies the behaviour of highly iterated functions is called the theory of dynamical systems. Traditionally, the functions that are going to be iterated-such as $F$ and $f$-are called maps. In particular, the map $f(11)$ is an example of a circle map (CM), i.e., a map from the circle $S^{1}$ to itself. The theory of CMs is a prominent part of theory of dynamical systems; it was initiated by Poincaré in the $1880 \mathrm{~s}$, and nowadays is a highly developed field of mathematics with many physical applications.

The relationship between the time advance map $F: \mathbb{R} \rightarrow$ $\mathbb{R}$ and the CM $f: S^{1} \rightarrow S^{1}$ is shown pictorially in figure 2 . Note that although $f$ looks discontinuous in the figure, it is continuous as a function on the circle $S^{1}$ because of the identifications of 0 and 1 shown in the figure with dotted lines. The map $F$ is called a lift of $f$, while $f$ is sometimes called the projection of $F$. Clearly, $F$ determines $f$ uniquely; on the other hand, each $\mathrm{CM} f$ has infinitely many lifts that differ by an additive integer constant (in our case, however, the lift $F$ is defined uniquely by (9)).

\section{Circle maps and wavepacket formation}

In this section we collect some facts about the dynamics, i.e., the behaviour of the high iterates $f^{n}$, of CMs. For more information the reader can consult the introductory expositions in [33, chapter 4] or [16, section 1.14], or the more sophisticated treatments in [41, chapters 11 and 12], [15, chapter I]. Section 3 of our paper [47] contains a selection of mathematical facts adapted to the problem of the resonator. In section 3.3 we will give an interpretation of the mathematical results in terms of the asymptotic behaviour of the field in the resonator.

\subsection{Circle maps-basic definitions}

By a circle map (CM), we will always mean a smooth $\left(C^{\infty}\right)$ invertible map of the circle whose inverse is also smooth-this is exactly the class of CMs that correspond to motions of the boundary $a$ satisfying the conditions from section 2.1. For the map $f$ to be invertible, we have to assume that the cavity is not too long, or, more concretely, that $F(t)-t<1$ (for which it is enough to assume that $a(t)<\frac{1}{2}$ for all $t$ ). This condition only helps to avoid clumsy sentences, and is not a restriction of the generality-our ideas can be easily applied mutatis mutandis to the case of a longer cavity.

The most important characteristic of a CM is its rotation number defined as the 'average amount of rotation':

$$
\tau(f) \equiv \tau(F):=\lim _{n \rightarrow \infty} \frac{F^{n}(t)-t}{n}
$$

(with the above restriction on the length of the cavity, $\tau(f) \in$ $[0,1))$. It can be proved that $\tau(f)$ always exists and does not depend on the value of $t$ in (12).

An orbit of a point $t \in S^{1}$ is the set $\left\{f^{n}(t)\right\}_{n=0}^{\infty}$ of all future (i.e., for $n \geqslant 0$ ) iterates of $t$. If for some point $t^{*} \in S^{1}$ there exists an integer $q$ such that $f^{q}\left(t^{*}\right)=t^{*}$, then we say that $t^{*}$ is a periodic point of period $q$ (or a $q$-periodic point) and call the orbit $\left\{f^{n}\left(t^{*}\right)\right\}_{n=0}^{q-1}$ of this point a $q$-periodic orbit.

The simplest example of a CM is the rigid rotation $r_{\sigma}$ : $S^{1} \rightarrow S^{1}$ (where $\sigma \in[0,1)$ ) defined through its lift $R_{\sigma}$,

$$
\begin{array}{cc}
R_{\sigma}(t):=t+\sigma, & t \in \mathbb{R}, \\
r_{\sigma}(t):=\{t+\sigma\}, & t \in S^{1} .
\end{array}
$$

Clearly, $\tau\left(r_{\sigma}\right)=\sigma$. The dynamics of $r_{\sigma}$ is very simple:

- if $\sigma$ is a rational number, i.e., $\sigma=p / q$ for some integers $p$ and $q$ (we will always assume that $p$ and $q$ do not have common factors), then after $q$ iterations any point $t \in S^{1}$ returns to its initial position, having traversed the circle $p$ times; i.e., each point $t \in S^{1}$ is a $q$-periodic point:

$$
\begin{gathered}
R_{p / q}^{q}(t)=t+p \quad \forall t \in \mathbb{R}, \\
r_{p / q}^{q}(t)=t \quad \forall t \in S^{1} ;
\end{gathered}
$$

- if $\sigma$ is not a rational number, then the orbit $\left\{r_{\sigma}^{n}(t)\right\}_{n=0}^{\infty}$ of any point $t \in S^{1}$ fills the circle densely, and will never return to the initial point $t$; thus, in this case there are no periodic orbits.

\subsection{Phase locking, Arnol'd tongues, the devil's staircase}

Here we will describe in detail the case of a general CM $f$ with a rational rotation number, $\tau(f)=p / q$, in which case 

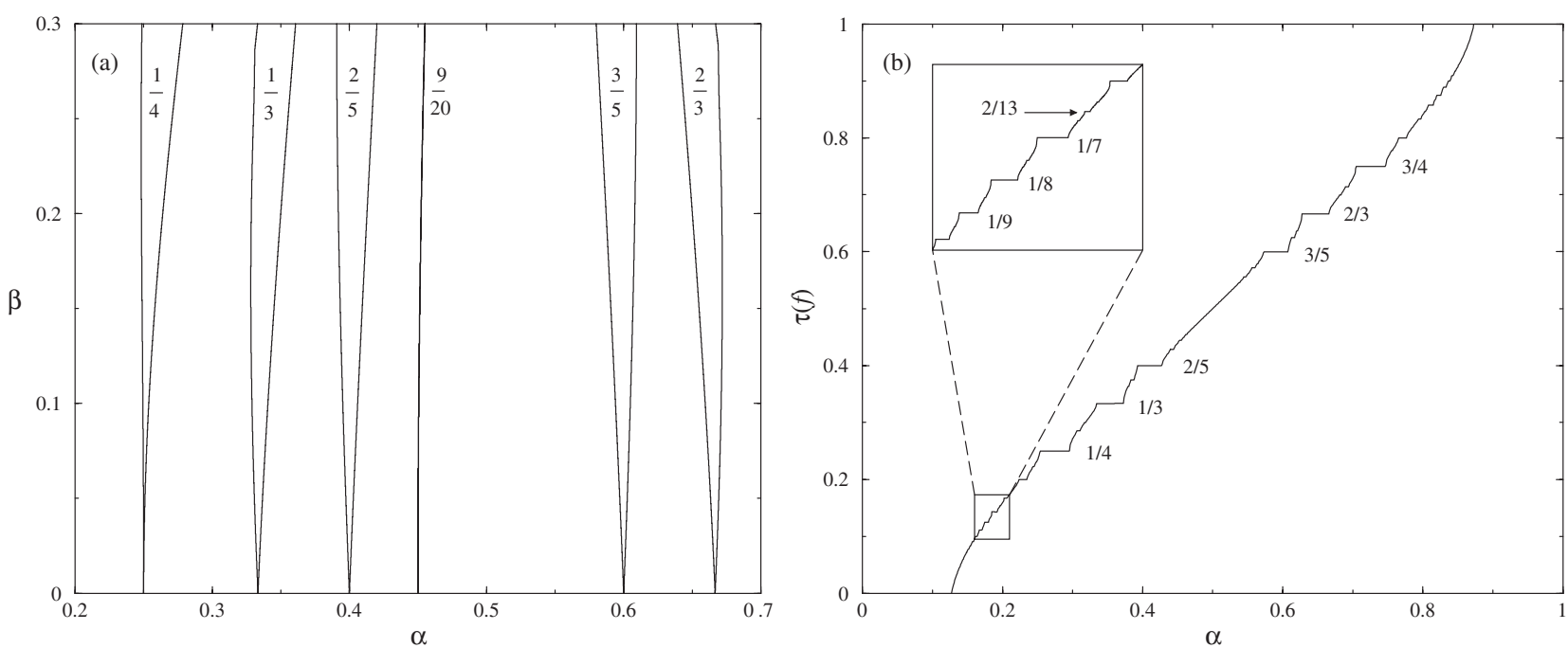

Figure 3. (a) Arnol'd tongues with rotation numbers $1 / 4,1 / 3,2 / 5,9 / 20,3 / 5$, and $2 / 3$ for the CM $f$ corresponding to motion of the boundary (2) with $\gamma=0.7$. (b) A graph of the rotation number $\tau(f)$ for (2) with $\beta=0.4, \gamma=0.7$.

the map $f$ is said to be phase locked (frequency locked, mode locked).

If $\tau(f)=p / q$, then generically $f$ has an attracting $q$-periodic orbit $\left\{t_{j}^{(\mathrm{a})}\right\}_{j=1}^{q}$, and a repelling $q$-periodic orbit $\left\{t_{j}^{(\mathrm{r})}\right\}_{j=1}^{q}$. 'Attracting' means that the orbit of each point $t \in S^{1}$ that is not one of the of the repelling periodic points $\left\{t_{j}^{(\mathrm{r})}\right\}_{j=1}^{q}$ tends asymptotically to the attracting periodic orbit $\left\{t_{j}^{(\mathrm{a})}\right\}_{j=1}^{q}$. The repelling periodic orbit 'repels' the iterates of $f$; it is an attracting periodic orbit for the inverse map $f^{-1}$ (which is also a CM). The attracting and repelling periodic orbits of the $\mathrm{CM} f$ give rise to attracting and repelling characteristics of the wave equation, and to formation of wavepackets (see section 3.3).

A question very important for the physics of the problem is how 'generic' the case of phase locking is. In figure 3(a) we show in the $(\alpha, \beta)$ plane the regions of values of the parameters $\alpha$ and $\beta$ of the motion of the mirror that correspond to phase locking of several rotation numbers $\tau(f)$. These 'phase locked' regions in the $(\alpha, \beta)$ plane are called Arnol'd tongues in honor of Arnol'd who studied them in his famous paper on CMs [3]. The Arnol'd tongue corresponding to a $p / q$ phase locking emanates (i.e., starts as $\beta \rightarrow 0^{+}$) from $\alpha=p / q$, and becomes thicker as $\beta$ increases. Tongues with large $p$ and $q$ are very thin (see the $9 / 20$ tongue in figure 3(a)).

Another illustration of the abundance of phase locking is the graph of the rotation number $\tau(f)$ versus $\alpha$ (all other parameters fixed), shown in figure 3(b). It can be proved that this function is continuous, and it is locally constant if $\tau(f)$ is rational, and strictly increasing if $\tau(f)$ is irrational, i.e., the graph of such a function contains infinitely many densely interspersed horizontal pieces, each corresponding to a particular type of phase locking. Such a graph is called a devil's staircase. Several of the horizontal pieces in the figure are labelled with $p / q$ showing the type of phase locking. The behaviour of the widths of the Arnol'd tongues as $\beta \rightarrow 0^{+}$is studied by Jonker [40] and Davie [13].

It is worth noting that, in some sense, phase locking is more 'generic' than the unlocked case. Namely, if $\tau(f)$ is irrational, then there exists an arbitrarily small smooth perturbation of $f$ such that the perturbed map is phase locked. On the other hand, if $\tau(f)=p / q$, then the parameter values are either strictly inside or on the boundary of the $q / p$ Arnol'd tongue. If the parameters are strictly inside the tongue, then a small enough (but otherwise arbitrary) smooth perturbation will not change its rotation number, i.e., the perturbed map will have rotation number $p / q$. Practically, however, one should not forget that the width of the Arnol'd tongues decreases fast when $p$ and $q$ increase or when $\beta$ decreases.

If the $\mathrm{CM} f$ is in $p / q$ phase locking and the parameters are strictly inside the $p / q$ tongue, $f$ has an attracting $q$-periodic orbit and a repelling one. In this case, if $t^{*}$ belongs to the attracting periodic orbit, then $f^{q}\left(t^{*}\right)=t^{*}$ and $f^{\prime}\left(t^{*}\right)<1$. In the following, we will say that a phase locking is 'generic' if the parameters of the CM are strictly inside the Arnol'd tongue, in which case there exist an attracting and a repelling orbit. If the parameters of the map are on the boundary of the Arnol'd tongue (i.e., at some end on the corresponding horizontal piece of the graph in figure 3(b)), then there exists a $q$-periodic orbit which is neither attracting, nor repelling; in this case $f^{\prime}\left(t^{*}\right)=1$ where $t^{*}$ is a $q$-periodic point.

When $\beta$ reaches the critical value at which the boundary is moving at the speed of light at some moment in each period (i.e., if $\left|a^{\prime}(t)\right|=1$ for some $t$ ), then the total length of the phase locking intervals (i.e., of the horizontal pieces in figure 3(b)) becomes equal to 1, which means physically that the probability of phase locking is 1 . This mathematical problem is studied numerically by Jensen et al [38] and Lanford [43], and proved rigorously for general CMs by Graczyk and Świątek [32].

\subsection{The derivative of the circle map, the Doppler factor, and the formation of wavepackets}

Now we translate the mathematical facts about the dynamics of the $\mathrm{CM} f(11)$ and the time advance map $F(9)$ into asymptotic properties of the field in the cavity.

If a particular characteristic is reflected by the stationary mirror at time $t$, then the times of the subsequent reflections 
from the same mirror are given by $F^{n}(t)(n=1,2, \ldots)$; the phases (7) of the motion of the mirror at these times are given by the iterates of the corresponding $\mathrm{CM}, f^{n}(\{t\})$. If $f$ is phase locked with rotation number $\tau(f)=p / q$, then, generically, there exists an attracting periodic orbit $\left\{t_{j}^{(\mathrm{a})}\right\}_{j=1}^{q}$ which attracts the iterates of any point in $S^{1}$ (except the repelling periodic points) under the map $f$. Physically, each attracting periodic point $t_{j}^{(\mathrm{a})} \in S^{1}$ of the CM $f$ corresponds to an infinite sequence of times of the form $n+t_{j}^{(\mathrm{a})}$, where $n$ is any integer, such that the characteristics that are reflected from the stationary mirror at these times attract the nearby characteristics. We will call times of the form $n+t_{j}^{(\text {a) }}$ 'attracting $q$-periodic times', and the corresponding characteristics 'attracting $q$-periodic characteristics'. The presence of $q$ attracting periodic characteristics means physically that the field in the cavity develops (at most) $q$ wavepackets, whose widths decrease exponentially, and whose energies increase exponentially with time.

In the case of generic $p / q$ phase locking, the rate at which the characteristics get closer together is related to the first derivative of $F$ (or, equivalently, $f$ ), which in turn is related (according to equations (6), (8), (9)) to the Doppler factor $D(\Theta(t))$ at the time $\Theta(t)$ of the first reflection from the moving mirror after $t$ :

$$
F^{\prime}(t)=\frac{1+a^{\prime}(\Theta(t))}{1-a^{\prime}(\Theta(t))}=\frac{1}{D(\Theta(t))} .
$$

Asymptotically, the wavepackets are very narrow, so they are reflected from the moving mirror practically instantaneously, at times of the form $n+t_{j}^{(\mathrm{a})}$. The asymptotic 'cumulative' Doppler factor $\mathcal{D}_{q}$ over a sequence of $q$ consecutive reflections of the packet from the moving mirror (which takes total time $p$ according to the fact that $\left.F^{q}\left(t_{j}^{(\mathrm{a})}\right)=t_{j}^{(\mathrm{a})}+p\right)$ is equal to the product of Doppler factors at each of these $q$ reflections:

$$
\mathcal{D}_{q}:=\prod_{j=1}^{q} D\left(\Theta\left(t_{j}^{(\mathrm{a})}\right)\right)=\left[\prod_{j=1}^{q} F^{\prime}\left(t_{j}^{(\mathrm{a})}\right)\right]^{-1}=\left[\left(F^{q}\right)^{\prime}\left(t_{1}^{(\mathrm{a})}\right)\right]^{-1} .
$$

This formula holds exactly only in the case of classical EM field, when the motion of the mirror only amplifies the field through the Doppler effect at reflection. In section 4.2, we will see that in the quantum case the energy is not only amplified, but also created by the motion of the mirror, which introduces corrections to the rate of change of the energy.

In the case when the rotation number $\tau(f)$ is irrational, the field does not develop wavepackets, and its energy changes with time, but does not have a tendency towards steady growth or decay.

\section{Quantum effects in a periodically pulsating resonator}

\subsection{Moore's functional equation}

Moore [54] was the first to consider the problem of quantizing the electromagnetic field in a one-dimensional resonator with a moving wall. We leave out all the complications that he had to overcome in the development of a quantization scheme, and focus on one particular aspect of his treatment (adapting his equations to our approach). Let the motion of the mirror correspond to time advance map $F$ (9) of rotation number $\sigma$. Moore showed that in this case one has to look for an expansion of the field operator $A(t, x)$ in mode functions

$$
A_{k}(t, x)=\mathrm{e}^{-2 \pi \mathrm{i} k \frac{1}{\sigma} \Sigma(t-x)}-\mathrm{e}^{-2 \pi \mathrm{i} k \frac{1}{\sigma} \Sigma(t+x)},
$$$$
k=1,2,3, \ldots,
$$

where the function $\Sigma: \mathbb{R} \rightarrow \mathbb{R}$ satisfies Moore's functional equation

$$
\Sigma(t+a(t))=\Sigma(t-a(t))+\sigma
$$

which ensures that $A_{k}(t, x)$ satisfy the Dirichlet BCs (5) with $c_{1}=c_{2}=0$. This equation can be rewritten in terms of the map $F$ as $\Sigma \circ F(t)=\Sigma(t)+\sigma$, or, equivalently, as

$$
\Sigma \circ F=R_{\sigma} \circ \Sigma
$$

(where $R_{\sigma}(13)$ is the rigid rotation by $\sigma$ ), and interpreted as the fact that the value of $\Sigma$ changes between two consecutive reflections from the stationary mirror by $\sigma$. This implies, in particular, that if for some particular value $\bar{t}$ we know the values of $\Sigma(t)$ in the interval $[\bar{t}, F(\bar{t}))$, then we can reconstruct the function $\Sigma$ for all $t \in \mathbb{R}$ by using (15).

Moore's functional equation is easy to solve numerically. Let us assume that before $t=0$, the two mirrors were at rest, and at $t=0$ the right mirror started moving:

$$
a(t)= \begin{cases}\frac{\alpha}{2}, & t \leqslant 0 \\ \frac{\alpha}{2}+\frac{\beta}{2 \pi}\left(1-\mathrm{e}^{-t^{4}}\right) \sin (2 \pi t & \\ \left.+\gamma(\sin 4 \pi t)^{2}\right), & t>0 .\end{cases}
$$

The motion of the boundary for $t>0$ is very similar to the one in (2); we have used the factor $1-\mathrm{e}^{-t^{4}}$ to smooth out the transition (i.e., to ensure that $\Sigma^{\prime}, \Sigma^{\prime \prime}$, and $\Sigma^{\prime \prime \prime}$ are continuous, the reason for which will become clear in section 4.2). The factor $\left(1-\mathrm{e}^{-t^{4}}\right)$ tends to 1 very quickly as $t$ grows, so it does not affect the asymptotic behaviour of the system. If the mirrors are stationary, it is natural to take the function $\Sigma(t)$ to be linear, so for $t \in[-\alpha, 0)$, we take $\Sigma(t)=\frac{\sigma}{\alpha} t+$ const (the constant is immaterial since only the derivatives of $\Sigma$ have physical meaning), and then use (15) to find $\Sigma(t)$ for $t \geqslant 0$.

Now we will apply our knowledge about the dynamics of the CM $f$-and, hence, about the time advance map $F$ to draw conclusions about the asymptotic behaviour of the function $\Sigma$, which will allow us to make predictions about the long-time behaviour of the energy density of the field. Since the case of a rational $\sigma=p / q$ is especially interesting because of the occurrence of resonant phenomena (phase locking), we focus on this case in the rest of this subsection. In the case of a generic $p / q$ phase locking, the CM $f$ has an attracting and a repelling $q$-periodic orbit. Let $t_{1}^{(\mathrm{a})}, \ldots, t_{q}^{(\mathrm{a})}$ be a sequence of $q$ consecutive attractive $q$-periodic times, and let the ordering be such that

$$
t_{1}^{(\mathrm{a})} \stackrel{F}{\mapsto} t_{2}^{(\mathrm{a})} \stackrel{F}{\mapsto} \cdots \stackrel{F}{\mapsto} t_{q}^{(\mathrm{a})} \stackrel{F}{\mapsto} t_{1}^{(\mathrm{a})}+p
$$

(this implies that the fractional parts of these times satisfy $\left.\left\{t_{1}^{(\mathrm{a})}\right\} \stackrel{f}{\mapsto} \cdots \stackrel{f}{\mapsto}\left\{t_{q}^{(\mathrm{a})}\right\} \stackrel{f}{\mapsto}\left\{t_{1}^{(\mathrm{a})}\right\}\right)$. If $n$ is a positive integer, then (15) iterated $n q$ times reads

$$
\Sigma \circ F^{n q}(t)=\Sigma(t)+n p .
$$



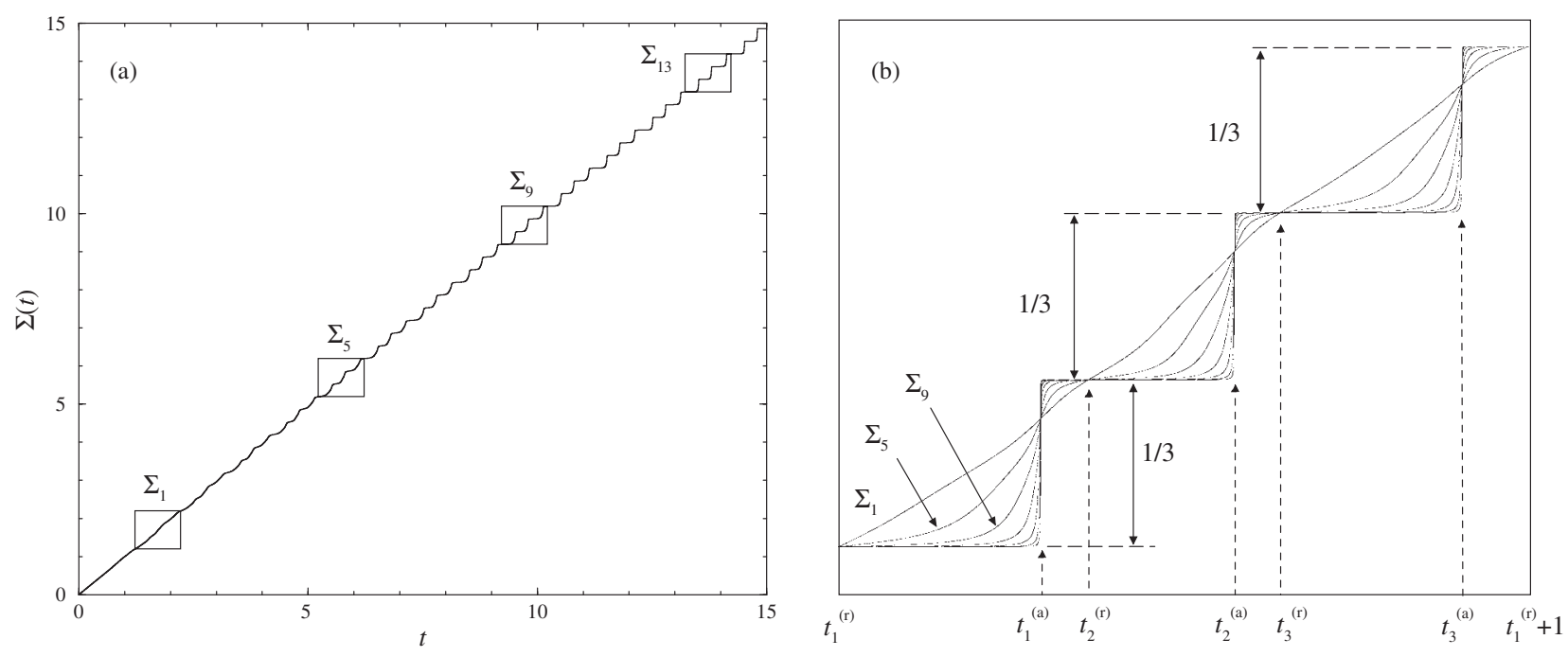

Figure 4. (a) Development of the staircase-like structure of the function $\Sigma$ for motion of the mirror given by (16) with $\alpha=0.34, \beta=0.2$, $\gamma=0.3$, corresponding to $1 / 3$ phase locking. (b) Graphs of the functions $\Sigma_{n}(17), n=1,5,9,13,17,21,25$, for the same parameter values.

Using that $F^{n q}\left(t_{1}^{(\mathrm{r})}\right)=t_{1}^{(\mathrm{r})}+n p$, we obtain

$$
\Sigma\left(t_{1}^{(\mathrm{r})}+n p\right)=\Sigma\left(t_{1}^{(\mathrm{r})}\right)+n p .
$$

This allows us to define a sequence of functions (for $n=0,1$, ...)

$$
\begin{gathered}
\Sigma_{n}:\left[t_{1}^{(\mathrm{r})}, t_{1}^{(\mathrm{r})}+n p\right) \rightarrow\left[\Sigma\left(t_{1}^{(\mathrm{r})}\right), \Sigma\left(t_{1}^{(\mathrm{r})}\right)+n p\right) \\
\Sigma_{n}(t):=\Sigma(t+n p)-n p
\end{gathered}
$$

and study the behaviour of $\Sigma(t)$ for very large $t$ by analysing the behaviour of $\Sigma_{n}(t)\left(\right.$ where $\left.t \in\left[t_{1}^{(\mathrm{r})}, t_{1}^{(\mathrm{r})}+n p\right)\right)$ for $n \rightarrow \infty$. The graph of $\Sigma$ can be assembled from translations of the graphs of $\Sigma_{n}$ as shown in figure 4(a), for parameters corresponding to $1 / 3$ phase locking. In figure $4(\mathrm{~b})$, we show the graphs of several $\Sigma_{n}$ for the same parameter values.

In the case of generic $p / q$ phase locking, the times of reflection, $t, F(t), F^{2}(t), \ldots$, of a particular characteristic from the stationary mirror accumulate at the attracting periodic times (of the form $n+t_{j}^{(\mathrm{a})}$ ), while the values of $\Sigma$ for two consecutive reflections differ by the constant value $\sigma$. This difference between the behaviour of the arguments and the values of the function $\Sigma(\operatorname{cf}(15))$ explains the occurrence of exactly $q$ steep parts of the graphs of $\Sigma_{n}$ at $t=t_{j}^{(\mathrm{a})}(j=1, \ldots, q)$, and $q$ almost horizontal parts for large $n$. Physically, the steep parts correspond to the times of reflection of the packets of the field; the 'widths' of these packets are given approximately by (cf (14))

$$
\Delta_{j}^{(n)} \sim \text { const } \times\left[\left(F^{q}\right)^{\prime}\left(t_{1}^{(\mathrm{a})}\right)\right]^{n}=\frac{\text { const }}{\mathcal{D}_{q}^{n}} .
$$

\subsection{The energy of the quantum field}

Fulling and Davies [30] computed the energy density of the quantum field in a one-dimensional cavity with one stationary and one moving mirror using the 'point-splitting' method of DeWitt [17]. They found that the regularized energy density in the space between the mirrors (i.e., the energy minus an infinite constant) is a superposition of the energies of left- and right-propagating disturbances:

$$
\left\langle T_{00}(t, x)\right\rangle_{\mathrm{reg}}=-\frac{1}{24 \pi}[\Phi(t+x)+\Phi(t-x)],
$$

where

$$
\Phi(\xi)=\mathscr{S}_{\Sigma}(\xi)+\frac{2 \pi^{2}}{\sigma^{2}}\left[\Sigma^{\prime}(\xi)\right]^{2},
$$

and $\mathscr{S}_{\Sigma}$ is the Schwarzian derivative of the function $\Sigma$, defined as

$$
\mathscr{S}_{\Sigma}(z):=\frac{\Sigma^{\prime \prime \prime}(z)}{\Sigma^{\prime}(z)}-\frac{3}{2}\left[\frac{\Sigma^{\prime \prime}(z)}{\Sigma^{\prime}(z)}\right]^{2} .
$$

The Schwarzian derivative is a remarkable (highly nonlinear!) differential operator, first introduced in complex analysis. If $\phi$ is a complex analytic function, then the vanishing of $\mathscr{S}_{\phi}$ is a necessary and sufficient condition for $\phi$ to be a Möbius (i.e., fractional linear) transformation, $M(z)=\frac{a z+b}{c z+d}$, where $a d-b c \neq 0$ (see, e.g., [57, chapter V]). The Schwarzian derivative is invariant with respect to a composition with a Möbius transformation, $\mathscr{S}_{M \circ F}=\mathscr{S}_{F}$, which follows from the identity

$$
\mathscr{S}_{G \circ H}(z)=\mathscr{S}_{G}(H(z))\left[H^{\prime}(z)\right]^{2}+\mathscr{S}_{H}(z) .
$$

The Schwarzian derivative appears in many branches of mathematics-dynamical systems [59], [15, chapter 1], [31], Lorentzian geometry $[42,27,28,60]$, the theory of differential equations [35, chapter 10], integrable systems theory [5], among many others. Even more interestingly, the Schwarzian derivative is widely used as a tool in the theory of CMs (see [34, 32]).

One can use the property (15) and the composition rule (20) to predict the long-time behaviour of the energy density (18). To this end, differentiate both sides of $\Sigma \circ F^{j}=$ $R_{j \sigma} \circ \Sigma$ (which is (15) iterated $j$ times) to obtain

$$
\Sigma^{\prime}\left(F^{j}(t)\right)=\frac{\Sigma^{\prime}(t)}{\left(F^{j}\right)^{\prime}(t)} .
$$


On the other hand, taking the Schwarzian derivative of the same relationship and using (20) with $G=\Sigma, H=F^{j}$, we have

$$
\mathscr{S}_{\Sigma}\left(F^{j}(t)\right)=\frac{1}{\left[\left(F^{j}\right)^{\prime}(t)\right]^{2}}\left[\mathscr{S}_{\Sigma}(t)-\mathscr{S}_{F^{j}}(t)\right] .
$$

These expressions yield

$$
\Phi\left(F^{j}(t)\right)=\frac{1}{\left[\left(F^{j}\right)^{\prime}(t)\right]^{2}}\left[\Phi(t)-\mathscr{S}_{F^{j}}(t)\right] .
$$

Using this equation, we can compute the energy of the field at an arbitrary space-time point if the function $\Sigma(t)$ is known for $t \in[\bar{t}, F(\bar{t}))$ where $\bar{t}$ is an arbitrary value; in particular, if for $t<0$ the mirrors are at rest, we can take $\Sigma(t)$ as in the discussion after (16) (there we used the smoothing factor $1-\mathrm{e}^{-t^{4}}$ because $\mathscr{S}_{\Sigma}$ contains third derivatives of $\Sigma$ ). In the case of classical EM field, the evolution of the energy density is similar to (21) except for the term $\mathscr{S}_{F^{j}}(t)$, which corresponds to the purely quantum effect of creation of a field by the motion of the mirror. Of course, in the absence of an electromagnetic field in the cavity at $t=0$, the classical energy is zero for all $t \geqslant 0$, while the energy of the quantum field is non-zero for $t>0$ even if at $t=0$ it was zero.

The composition rule (20) implies

$$
\begin{aligned}
\mathscr{S}_{F^{j}}(t) & =\sum_{k=0}^{j-1} \mathscr{S}_{F}\left(F^{k}(t)\right)\left[\left(F^{k}\right)^{\prime}(t)\right]^{2} \\
& =\sum_{k=0}^{j-1} \mathscr{S}_{F}\left(F^{k}(t)\right) \prod_{i=0}^{k-1}\left[F^{\prime}\left(F^{i}(t)\right)\right]^{2},
\end{aligned}
$$

which allows us to rewrite equation (21) as

$$
\Phi\left(F^{j}(t)\right)=\frac{\Phi(t)}{\left[\left(F^{j}\right)^{\prime}(t)\right]^{2}}-\sum_{k=0}^{j-1} \frac{\mathscr{S}_{F}\left(F^{k}(t)\right)}{F^{\prime}\left(F^{j-1}(t)\right)^{2} \cdots F^{\prime}\left(F^{k}(t)\right)^{2}} .
$$

\subsection{The physical mechanism of the energy changes}

In this subsection, we give a transparent physical interpretation of the terms in the right-hand side of (22). Namely, the first term in the right-hand side of (22) is the initial energy density amplified in the $j$ reflections from the moving mirror between $t$ and $F^{j}(t)$, while the term with summation index $k$ in the sum corresponds to the field created due to the motion of the mirror at time $F^{k}(t)$ and subsequently amplified at each of the following reflections. To prove this, we will use the following result concerning the energy density emitted by a single moving mirror. Fulling and Davies [30] proved that if a perfect mirror is moving in vacuum according to $x=a(t)$, then the regularized energy density to the right of the mirror (i.e., for $x>a(t))$ is given by

$\left\langle T_{00}(t, x)\right\rangle_{\mathrm{reg}}=-\frac{1}{24 \pi} \mathscr{S}_{F}(t-x)=-\frac{1}{24 \pi} \mathscr{S}_{F}\left((\operatorname{Id}-a)\left(t_{\mathrm{e}}\right)\right)$,

where $F$ is given by (9), and $t_{\mathrm{e}}$ is the time of emission. To the left of the mirror (for $x<a(t)$ ), the energy density is given by

$\left\langle T_{00}(t, x)\right\rangle_{\mathrm{reg}}=-\frac{1}{24 \pi} \mathscr{S}_{\tilde{F}}(t+x)=-\frac{1}{24 \pi} \mathscr{S}_{\tilde{F}}\left((\mathrm{Id}+a)\left(t_{\mathrm{e}}\right)\right)$, where $\tilde{F}=(\operatorname{Id}-a) \circ(\operatorname{Id}+a)^{-1}$ is defined similarly to $F(9)$, but with $a$ replaced by $-a$. Similarly to (8), we define the function $\tilde{\Theta}=(\mathrm{Id}+a)^{-1}$.

To rewrite (23) in another form, we will need the following formulae:

$$
\mathscr{S}_{\operatorname{Id} \pm a}(\xi)=\frac{a^{\prime \prime \prime}(\xi)\left[ \pm 1+a^{\prime}(\xi)\right]-\frac{3}{2} a^{\prime \prime}(\xi)^{2}}{\left[1 \pm a^{\prime}(\xi)\right]^{2}},
$$

(which follows directly from (19)), and

$$
\mathscr{S}_{G}\left(G^{-1}(\xi)\right)=-\left[G^{\prime}\left(G^{-1}(\xi)\right)\right]^{2} \mathscr{S}_{G^{-1}}(\xi)
$$

(a consequence of (20)), which in turn implies

$$
\begin{aligned}
\mathscr{S}_{\tilde{\Theta}}\left((\operatorname{Id}+a)\left(t_{\mathrm{e}}\right)\right) & =-\tilde{\Theta}^{\prime}\left((\operatorname{Id}+a)\left(t_{\mathrm{e}}\right)\right)^{2} \mathscr{S}_{\mathrm{Id}+a}\left(t_{\mathrm{e}}\right) \\
& =-\frac{\mathscr{S}_{\mathrm{Id}+a}\left(t_{\mathrm{e}}\right)}{\left[1+a^{\prime}\left(t_{\mathrm{e}}\right)\right]^{2}} .
\end{aligned}
$$

Now we have

$$
\begin{aligned}
& \mathscr{S}_{\tilde{F}}\left((\operatorname{Id}+a)\left(t_{\mathrm{e}}\right)\right)=\mathscr{S}_{(\mathrm{Id}-a) \circ \tilde{\Theta}}\left((\operatorname{Id}+a)\left(t_{\mathrm{e}}\right)\right) \\
& =\left[\tilde{\Theta}^{\prime}\left((\operatorname{Id}+a)\left(t_{\mathrm{e}}\right)\right)\right]^{2} \mathscr{S}_{\mathrm{Id}-a}\left(t_{\mathrm{e}}\right)+\mathscr{S}_{\tilde{\Theta}}\left((\mathrm{Id}+a)\left(t_{\mathrm{e}}\right)\right) \\
& =\left[\tilde{\Theta}^{\prime}\left((\operatorname{Id}+a)\left(t_{\mathrm{e}}\right)\right)\right]^{2}\left[\mathscr{S}_{\mathrm{Id}-a}\left(t_{\mathrm{e}}\right)-\mathscr{S}_{\mathrm{Id}+a}\left(t_{\mathrm{e}}\right)\right] \\
& =-2 \frac{a^{\prime \prime \prime}\left(t_{\mathrm{e}}\right)\left[1-a^{\prime}\left(t_{\mathrm{e}}\right)^{2}\right]+3 a^{\prime}\left(t_{\mathrm{e}}\right) a^{\prime \prime}\left(t_{\mathrm{e}}\right)^{2}}{\left[1-a^{\prime}\left(t_{\mathrm{e}}\right)\right]^{4}\left[1+a^{\prime}\left(t_{\mathrm{e}}\right)\right]^{2}} .
\end{aligned}
$$

Now we will use (24) to understand the physical meaning of (22). For simplicity, we consider the evolution of the energy density along a characteristic which at time $t_{0}$ passes through the point $x_{0}$ while moving to the left. At time $t_{+}:=t_{0}+x_{0}$ this characteristic is reflected from the stationary mirror, then at time $\Theta\left(t_{+}\right)$it is reflected from the moving mirror; the next three reflections occur at times $F\left(t_{+}\right), \Theta\left(F\left(t_{+}\right)\right)$, and $F^{2}\left(t_{+}\right)$, respectively. Let $t_{1}$ be a time after $F^{2}\left(t_{+}\right)$but before the next reflection, and $x_{1}=t_{1}-F^{2}\left(t_{+}\right)$be the spatial coordinate of the characteristic at time $t_{1}$. For the energy density we obtain from (22)

$$
\begin{aligned}
& \left\langle T_{00}\left(t_{1}, x_{1}\right)\right\rangle_{\mathrm{reg}}=D\left(\Theta\left(F\left(t_{+}\right)\right)\right)^{2} D\left(\Theta\left(t_{+}\right)\right)^{2}\left\langle T_{00}\left(t_{0}, x_{0}\right)\right\rangle_{\mathrm{reg}} \\
& \quad+D\left(\Theta\left(F\left(t_{+}\right)\right)\right)^{2} \frac{1}{24 \pi} \frac{\mathscr{S}_{F}\left(t_{+}\right)}{F^{\prime}\left(t_{+}\right)^{2}}+\frac{1}{24 \pi} \frac{\mathscr{S}_{F}\left(F\left(t_{+}\right)\right)}{F^{\prime}\left(F\left(t_{+}\right)\right)^{2}} .
\end{aligned}
$$

The first term in the right-hand side of (25) is the initial energy density $\left\langle T_{00}\left(t_{0}, x_{0}\right)\right\rangle_{\text {reg }}$ amplified by the factor of $D\left(\Theta\left(F\left(t_{+}\right)\right)\right)^{2}=\left[F^{\prime}\left(t_{+}\right)\right]^{-2}$ at reflection from the moving mirror at time $\Theta\left(t_{+}\right)$, and by the factor of $D\left(\Theta\left(F\left(t_{+}\right)\right)\right)^{2}=$ $\left[F^{\prime}\left(F\left(t_{+}\right)\right)\right]^{-2}$ at the next reflection from the moving mirror at time $\Theta\left(F\left(t_{+}\right)\right)$.

The second term in the right-hand side of (25) is the energy density created at reflection of the characteristic from the moving mirror at time $\Theta\left(t_{+}\right)$, and consequently amplified by the factor of $D\left(\Theta\left(F\left(t_{+}\right)\right)\right)^{2}$ at the next reflection from the moving mirror. Indeed, we have

$$
\begin{aligned}
\mathscr{S}_{F}\left(t_{+}\right) & =\mathscr{S}_{(\mathrm{Id}+a) \circ \Theta}\left(t_{+}\right) \\
& =\left[\Theta^{\prime}\left(t_{+}\right)\right]^{2} \mathscr{S}_{\mathrm{Id}+a}\left(\Theta\left(t_{+}\right)\right)+\mathscr{S}_{\Theta}\left(t_{+}\right) \\
& =\left[\Theta^{\prime}\left(t_{+}\right)\right]^{2}\left[\mathscr{S}_{\mathrm{Id}+a}\left(\Theta\left(t_{+}\right)\right)-\mathscr{S}_{\mathrm{Id}-a}\left(\Theta\left(t_{+}\right)\right)\right] \\
& =\left.2 \frac{a^{\prime \prime \prime}(\xi)\left[1-a^{\prime}(\xi)^{2}\right]+3 a^{\prime}(\xi) a^{\prime \prime}(\xi)^{2}}{\left[1-a^{\prime}(\xi)\right]^{4}\left[1+a^{\prime}(\xi)\right]^{2}}\right|_{\xi=\Theta\left(t_{+}\right)} .
\end{aligned}
$$


Together with (24), this equality implies

$$
\begin{aligned}
& \frac{1}{24 \pi} \frac{\mathscr{S}_{F}\left(t_{+}\right)}{F^{\prime}\left(t_{+}\right)^{2}} \\
& =\left.\frac{1}{12 \pi} \frac{a^{\prime \prime \prime}(\xi)\left[1-a^{\prime}(\xi)^{2}\right]+3 a^{\prime}(\xi) a^{\prime \prime}(\xi)^{2}}{\left[1-a^{\prime}(\xi)\right]^{2}\left[1+a^{\prime}(\xi)\right]^{4}}\right|_{\xi=\Theta\left(t_{+}\right)} \\
& =-\frac{1}{24 \pi} \mathscr{S}_{\tilde{F}}\left((\operatorname{Id}+a)\left(\Theta\left(t_{+}\right)\right)\right),
\end{aligned}
$$

and a comparison with (26) proves the correctness of our interpretation of the term $\frac{1}{24 \pi} \frac{\mathscr{S}_{F}\left(t_{+}\right)}{F^{\prime}\left(t_{+}\right)^{2}}$.

Similarly, the third term in the right-hand side of (25) is the energy density created by the moving mirror at time $\Theta\left(F\left(t_{+}\right)\right)$.

This discussion elucidates the difference between the classical and the quantum cases - in the classical case the moving mirror amplifies the wavepackets by squeezing them, while in the quantum case the moving mirror not only amplifies the already existing wavepackets, but also creates a new field which is subsequently amplified at each reflection from the moving mirror.

\subsection{Resonant amplification in a periodically pulsating cavity}

Now we will consider the particular case of periodic motion of the mirror when the corresponding $\mathrm{CM} f$ has rational rotation number, $\tau(f)=\sigma=p / q$, i.e., is in $p / q$ phase locking. In this case the classical EM field in the cavity develops wavepackets whose number can be anywhere between 1 and $q$ depending on the initial conditions. The wavepackets become narrower with each reflection, and their energy increases with each reflection.

In the quantum case, the motion of the mirror itself creates energy which is subsequently concentrated in narrow wavepackets. If the acceleration of the mirror is not zero except at isolated times, then the mirror is emitting energy all the time, and the number of wavepackets developed is exactly $q$.

In the case of $p / q$ phase locking, the $\mathrm{CM} f$ generically has an attracting $q$-periodic orbit, which corresponds to the times of reflection of the attracting characteristics. If $t^{*}$ is such a time (i.e., if the fractional part of $t^{*}$ belongs to the attracting $q$-periodic orbit of $f$ ), then

$$
F^{q}\left(t^{*}\right)=t^{*}+p, \quad 0<\left(F^{q}\right)^{\prime}\left(t^{*}\right)<1,
$$

which implies that asymptotically the cumulative Doppler factor $\mathcal{D}_{q}=\left[\left(F^{q}\right)^{\prime}\left(t^{*}\right)\right]^{-1}$ (see (14)) is greater than 1 . In the case of $p / q$ phase locking, (22) implies (for any integer $n$ )

$$
\Phi\left(F^{n q}(t)\right)=\frac{\Phi(t)}{\left[\left(F^{n q}\right)^{\prime}(t)\right]^{2}}-\sum_{j=0}^{n-1} \frac{\mathscr{S}_{F^{q}}\left(F^{j q}(t)\right)}{\left[\left(F^{(n-j) q}\right)^{\prime}\left(F^{j q}(t)\right)\right]^{2}} .
$$

If $t^{*}$ is a time of reflection of an attracting characteristic, (27) yields

$$
\begin{aligned}
\Phi\left(t^{*}+n p\right) & =\mathcal{D}_{q}^{2 n}\left(\Phi\left(t^{*}\right)-\mathscr{S}_{F^{q}}\left(t^{*}\right) \sum_{j=0}^{n-1} \mathcal{D}_{q}^{-2 j}\right) \\
& =\mathcal{D}_{q}^{2 n}\left(\Phi\left(t^{*}\right)-\frac{1-\mathcal{D}_{q}^{-2 n}}{1-\mathcal{D}_{q}^{-2}} \mathscr{S}_{F^{q}}\left(t^{*}\right)\right) .
\end{aligned}
$$

The applicability of this expression is not restricted to times such as $t^{*}$-since asymptotically all characteristics are very

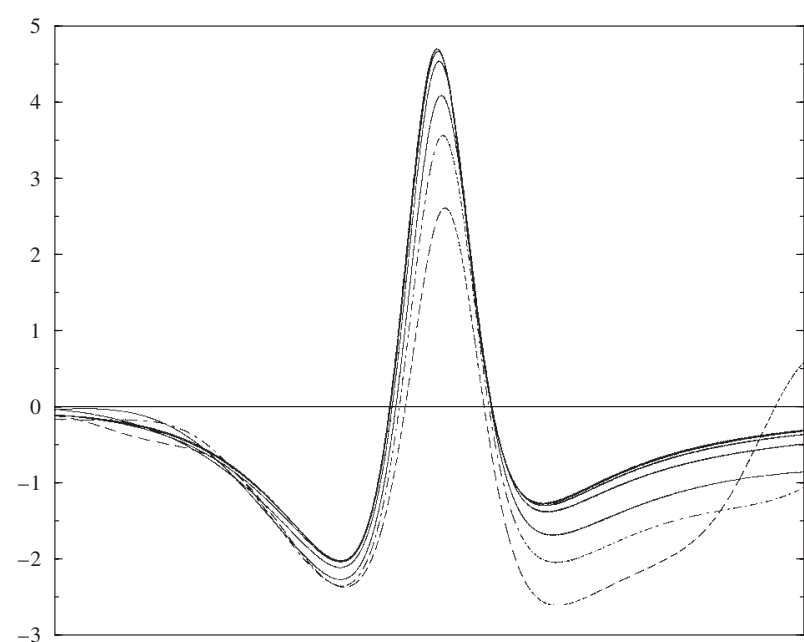

Figure 5. The rescaled 'shape' of a wavepacket at times 2 (dashed curve), 3 (dot-dashed curve), 4, 6, 8, 10, 12 (solid curves) (see the text).

close to the attracting ones, (28) gives approximately the asymptotic behaviour of the energy density of the wavepackets. Parenthetically, we remark that using this expression, one can prove that for motion of the mirror with parameters corresponding to the ends of the phase locking intervals (i.e., the ends of the horizontal parts of the graph in figure 3(b)), the energy density grows not exponentially, but polynomially:

$$
\lim _{\mathcal{D}_{q} \rightarrow 1^{+}} \Phi\left(t^{*}+n p\right)=\Phi\left(t^{*}\right)-n \mathscr{S}_{F^{q}}\left(t^{*}\right) .
$$

The energy of the $j$ th wavepacket at time $t$ is given by

$$
\begin{aligned}
\mathcal{E}_{j}(t) & =\int_{\Delta_{j}(t)}\left\langle T_{00}(t, x)\right\rangle_{\text {reg }} \mathrm{d} x \\
& =-\frac{1}{24 \pi} \int_{\Delta_{j}(t)}[\Phi(t-x)+\Phi(t+x)] \mathrm{d} x,
\end{aligned}
$$

where $\Delta_{j}(t)$ is the support of the $j$ th wavepacket at time $t$. Recalling that at reflection at time $t$ the width of the wavepacket decreases $D(t)$ times, we obtain that asymptotically the energy of the field in the cavity increases exponentially: for large $t$,

$$
\mathcal{E}_{\text {total }}(t) \sim \text { const } \times \mathcal{D}_{q}^{t / p} .
$$

The energy density of the wavepackets changes at each reflection from the moving mirror, but after appropriate rescaling, its 'shape' at times $n p$ as $n \rightarrow \infty$ tends to a some constant profile which depends on the motion of the mirror. In figure 5, we show the evolution of the 'shape' of a wavepacket for the same mirror motion as in figure 4(a). In the figure we show the rescaled energy density, $\mathcal{D}_{q}^{-2 n} \cdot\left\langle T_{00}(n, x)\right\rangle_{\text {reg }}$, on the vertical axis versus the shifted and rescaled spatial coordinate $\mathcal{D}_{q}^{n} \cdot\left(x-x_{n}^{*}\right)$ on the horizontal axis, at times $n$ for several values of $n$; here $x_{n}^{*}$ is the spatial coordinate of the attracting characteristic corresponding to this packet, at time $n$.

\section{Concluding remarks}

The power of the methods from the theory of dynamical systems is due to their generality. The predictions that we 
have made about the behaviour of the field in the cavity are applicable to any motion of the mirror, not only to particular examples. Within our approach, we gave a complete classification of the possible resonances (phase locking) in the system, predicted that, generically, small detuning does not destroy the resonance, gave a simple explanation of the squeezing of the wavepackets, interpreted the origin of the different contributions to the energy density in the cavity. We would also like to emphasize that our technique is nonperturbative.

In the case of resonance, the standard numerical methods for solving partial differential equations would be very difficult to apply because of the concentration of the field in narrow packets. The proposed method, however, relies on iterating one-dimensional maps, so resonances do not present any additional difficulty. The computer programs used to produce the pictures in this paper took minutes to run on a PC.

Our methodology easily generalizes (see our paper [58]) to the case of quasiperiodic motion of the mirror, and the case of two moving mirrors (studied previously by Ji et al [39], Dodonov [21], Dalvit and Mazzitelli [12], Li and Li [45]). Recently, similar ideas from dynamical systems have been applied to the study of waves in a fluid in a two-dimensional basin by Manders et al [51].

Interestingly, the behaviour of the field of the cavity (described by a partial differential equation) is easier to analyse than the behaviour of a particle bouncing back and forth between two perfectly reflecting walls (assuming that the reflections are perfectly elastic). The latter system, suggested by Fermi [29] as a possible mechanism for acceleration of the particles in cosmic rays, reveals a much richer dynamical behaviour (see, e.g., the book of Lichtenberg and Lieberman [46]).

There are many questions that deserve further study. An interesting question is whether the theory of dynamical systems can be applied to the case of a constant-length cavity filled with dielectric with changing properties. Another problem is the absence of resonances of a certain type (noticed in our paper [47] and discussed by Wȩgrzyn [61])—is it generic, and which resonances are forbidden? Can methods of dynamical systems theory be used to study the problem in higher dimensions? Can our methodology be applied to the recent suggestion by Jaffe and Scardicchio [37] of applying the methods of geometric optics to the study of the Casimir effect? Can similar methods be applied to other fields (see, e.g., the study of a classical massive field in a pulsating resonator by Dittrich and Duclos [18])?

The appearance of the Schwarzian derivative hints at possible deeper connections between the quantum problem of a moving mirror, partial differential equations, and dynamical systems.

\section{Acknowledgments}

I would like to express my gratitude to Rafael de la Llave who directed my attention to the classical aspects of the problem considered in this paper, which resulted in our paper [47] and motivated other collaborative research [48]. I had a very pleasant collaboration with him and John Vano on the generalization of our method to the case of quasiperiodic motion of the mirror [58]. I would like to acknowledge fruitful conversations with Gautam Bharali and Michael Bolt. My research was partially supported by the Rackham Faculty Fellowship of the Rackham Graduate School, University of Michigan.

Last but not least, I would like to thank the organizers of this Topical Issue, Gabriel Barton, Victor V Dodonov, and Vladimir I Man'ko, for the invitation to submit a paper.

\section{References}

[1] Alves D T, Farina C and Maia Neto P A 2003 Dynamical Casimir effect with Dirichlet and Neumann boundary conditions J. Phys. A: Math. Gen. 36 11333-42

[2] Andreata M A and Dodonov V V 2000 Energy density and packet formation in a vibrating cavity J. Phys. A: Math. Gen. 33 3209-23

[3] Arnol'd V I 1961 Small denominators. I. Mapping the circle onto itself Izv. Akad. Nauk SSSR Ser. Matem. 25 21-86

Arnol'd V I 1964 Izv. Akad. Nauk SSSR Ser. Matem. 28 479-80 (correction)

Arnol'd V I 1965 Am. Math. Soc. Transl. 246 213-84 (Engl. Transl.)

[4] Bordag M, Mohideen U and Mostepanenko V M 2001 New developments in the Casimir effect Phys. Rep. 353 1-205

[5] Burstall F, Pedit F and Pinkall U 2002 Schwarzian derivatives and flows of surfaces Differential Geometry and Integrable Systems (Tokyo, 2000) (Contemp. Math. vol 308) (Providence, RI: American Mathematical Society) pp 39-61 (Preprint math.DG/0111169)

[6] Cole C K and Schieve W C 1995 Radiation modes of a cavity with a moving boundary Phys. Rev. A 52 4405-15

[7] Cooper J 1980 Scattering of electromagnetic fields by a moving boundary: the one-dimensional case IEEE Trans. Antennas Propag. 28 791-5

[8] Cooper J 1993 Asymptotic behavior for the vibrating string with a moving boundary J. Math. Anal. Appl. 174 67-87

[9] Cooper J 1993 Long-time behavior and energy growth for electromagnetic waves reflected by a moving boundary IEEE Trans. Antennas Propag. 41 1365-70

[10] Cooper J and Koch H 1995 The spectrum of a hyperbolic evolution operator J. Funct. Anal. 133 301-28

[11] Crocce M, Dalvit D A R and Mazzitelli F D 2002 Quantum electromagnetic field in a three-dimensional oscillating cavity Phys. Rev. A 66033811

[12] Dalvit D A R and Mazzitelli F D 1999 Creation of photons in an oscillating cavity with two moving mirrors Phys. Rev. A 59 3049-59

[13] Davie A M 1996 The width of Arnold tongues for the sine circle map Nonlinearity 9 421-32

[14] Davies P C W and Fulling S A 1977 Quantum vacuum energy in two dimensional space-times Proc. R. Soc. A 354 59-77

[15] de Melo W and van Strien S 1993 One-Dimensional Dynamics (Berlin: Springer)

[16] Devaney R L 1989 An Introduction to Chaotic Dynamical Systems 2nd edn (Redwood City, CA: Addison-Wesley)

[17] DeWitt B S 1975 Quantum field theory in curved space-time Phys. Rep. 19 295-357

[18] Dittrich J and Duclos P 2002 Massive scalar field in a one-dimensional oscillating region J. Phys. A: Math. Gen. 35 8213-30

[19] Dittrich J, Duclos P and Gonzalez N 1998 Stability and instability of the wave equation solutions in a pulsating domain Rev. Math. Phys. 10 925-62

[20] Dittrich J, Duclos P and Šeba P 1994 Instability in a classical periodically driven string Phys. Rev. E 49 3535-8

[21] Dodonov V V 1998 Resonance photon generation in a vibrating cavity J. Phys. A: Math. Gen. 31 9835-54

[22] Dodonov V V 2001 Nonstationary Casimir effect and analytical solutions for quantum fields in cavities with 
moving boundaries Contemporary Optics and Electrodynamics, Part 1. Advances in Chemical Physics vol 119, ed M Evans (New York: Wiley) pp 309-94 (Preprint quant-ph/0106081)

[23] Dodonov V V and Andreata M A 1999 Squeezing and photon distribution in a vibrating cavity J. Phys. A: Math. Gen. 32 6711-26

[24] Dodonov V V and Klimov A B 1996 Generation and detection of photons in a cavity with a resonantly oscillating boundary Phys. Rev. A 53 2664-82

[25] Dodonov V V, Klimov A B and Man'ko V I 1990 Generation of squeezed states in a resonator with a moving wall Phys. Lett. A 149 225-8

[26] Dodonov V V, Klimov A B and Nikonov D E 1993 Quantum phenomena in resonators with moving walls J. Math. Phys. 34 2742-56

[27] Duval C and Guieu L 2000 The Virasoro group and Lorentzian surfaces: the hyperboloid of one sheet J. Geom. Phys. 33 103-27

[28] Duval C and Ovsienko V Yu 2000 Lorentz world lines and the Schwarzian derivative Funktsional. Anal. i Prilozhen. 34 69-72

Duval C and Ovsienko V Yu 2000 Funct. Anal. Appl. 34 135-7 (Engl. Transl.)

[29] Fermi E 1949 On the origin of the cosmic radiation Phys. Rev. $2751169-74$

[30] Fulling S A and Davies P C W 1976 Radiation from a moving mirror in two dimensional space-time: conformal anomaly Proc. R. Soc. A 348 393-414

[31] Graczyk J, Sands D and Świątek G 2001 La dérivée Schwarzienne en dynamique unimodale C. R. Acad. Sci. 332 329-32

[32] Graczyk J and Świątek G 1996 Critical circle maps near bifurcation Commun. Math. Phys. 176 227-60

[33] Hasselblatt B and Katok A 2003 A First Course in Dynamics: With a Panorama of Recent Developments (Cambridge: Cambridge University Press)

[34] Herman M-R 1985 Simple proofs of local conjugacy theorems for diffeomorphisms of the circle with almost every rotation number Bol. Soc. Brasil. Mat. 16 45-83

[35] Hille E 1976 Ordinary Differential Equations in the Complex Domain (New York: Wiley)

[36] Jaekel M-T and Reynaud S 1992 Motional Casimir force J. Physique I 2 142-65

[37] Jaffe R L and Scardicchio A 2004 Casimir effect and geometric optics Phys. Rev. Lett. 92070402

[38] Jensen M H, Bak P and Bohr T 1984 Transition to chaos by interaction of resonances in dissipative systems. I. Circle maps Phys. Rev. A 30 1960-9

[39] Ji J-Y, Jung H-H and Soh K-S 1998 Interference phenomena in the photon production between two moving mirrors Phys. Rev. A 57 4952-5

[40] Jonker L B 1990 The scaling of Arnol'd tongues for differentiable homeomorphisms of the circle Commun. Math. Phys. 129 1-25

[41] Katok A and Hasselblatt B 1995 Introduction to the Modern Theory of Dynamical Systems (Cambridge: Cambridge University Press)

[42] Kostant B and Sternberg S 1988 The Schwartzian [Schwarzian] derivative and the conformal geometry of the Lorentz hyperboloid Quantum Theories and Geometry (Les Treilles, 1987) (Math. Phys. Stud. vol 10) (Dordrecht: Kluwer-Academic) pp 113-25
[43] Lanford O E III 1985 A numerical study of the likelihood of phase locking Physica D 14 403-8

[44] Law C K 1994 Resonance response of the quantum vacuum to an oscillating boundary Phys. Rev. Lett. 73 1931-4

[45] Li L and Li B-Z 2002 Numerical solutions of the generalized Moore's equations for a one-dimensional cavity with two moving mirrors Phys. Lett. A 300 27-32

[46] Lichtenberg A J and Lieberman M A 1992 Regular and Chaotic Dynamics 2nd edn (Berlin: Springer)

[47] de la Llave R and Petrov N P 1999 Theory of circle maps and the problem of one-dimensional optical resonator with a periodically moving wall Phys. Rev. E $596637-51$

[48] de la Llave R and Petrov N P 2002 Regularity of conjugacies between critical circle maps: an experimental study Exp. Math. 11 219-41

[49] Maclay G J and Forward R L 2004 A gedanken spacecraft that operates using the quantum vacuum (dynamic Casimir effect) Found. Phys. 34 477-500

[50] Maia Neto P A 2003 Vacuum radiation pressure on moving mirrors J. Phys. A: Math. Gen. 27 2167-80

[51] Manders A M M, Duistermaat J J and Maas L R M 2003 Wave attractors in a smooth convex enclosed geometry Physica D 186 109-32

[52] Méplan O and Gignoux C 1996 Exponential growth of a wave in a $1 \mathrm{D}$ vibrating cavity: application to the quantum vacuum Phys. Rev. Lett. 76 408-10

[53] Milton K A 2001 The Casimir Effect: Physical Manifestations of Zero-Point Energy (River Edge, NJ: World Scientific)

[54] Moore G T 1970 Quantum theory of the electromagnetic field in a variable-length one-dimensional cavity J. Math. Phys. 11 2679-91

[55] Mostepanenko V M and Trunov N N 1997 The Casimir Effect and Its Applications (Oxford: Clarendon)

[56] Mundarain D F and Maia Neto P A 1998 Quantum radiation in a cavity with moving mirrors Phys. Rev. A 57 1379-90

[57] Nehari Z 1952 Conformal Mapping (New York: McGraw-Hill)

[58] Petrov N P, de la Llave R and Vano J A 2003 Torus maps and the problem of one-dimensional optical resonator with a quasiperiodically moving wall Physica D $180140-84$

[59] Singer D 1978 Stable orbits and bifurcation of maps of the interval SIAM J. Appl. Math. 35 260-7

[60] Singer D A 2001 Diffeomorphisms of the circle and hyperbolic curvature Conform. Geom. Dyn. 5 1-5 (electronic)

[61] Wȩgrzyn P 2004 Parametric resonance in a vibrating cavity Phys. Lett. A 322 263-9

[62] Wu Y, Chan K W, Chu M-C and Leung P T 1999 Radiation modes of a cavity with a resonantly oscillating boundary Phys. Rev. A 59 1662-6

[63] Yamaguchi M 1997 Quasiperiodic motions of vibrating string with periodically moving boundaries J. Differ. Eqns 135 $1-15$

[64] Yamaguchi M 1998 Periodic motions of vibrating string with a periodically moving boundary Discrete Contin. Dynam. Systems Added volume II 303-14

[65] Yamaguchi M 2002 Periodic solutions of nonlinear equations of string with periodically oscillating boundaries Funkcial. Ekvac. 45 397-416

[66] Yamaguchi M and Yoshida H 2000 Nonhomogeneous string problem with periodically moving boundaries Operator Theory and Its Applications (Winnipeg, MB, 1998) (Providence, RI: American Mathematical Society) pp 565-74 